\title{
REGULACIÓN EMOCIONAL Y FUNCIONAMIENTO SOCIAL EN PACIENTES CON ESQUIZOFRENIAd
}

\author{
Dulce Romero-Ayuso $^{\mathrm{a}}{ }^{1} \mathbb{1}$, \& José García $\operatorname{Arenas}^{\mathrm{b}}{ }^{(\mathbb{C}}{ }^{2}$ \\ Universidad de Granada, Granada, España. ${ }^{\text {a }}$ \\ Centro de Salud Mental de Lorca, Murcia, España. ${ }^{\text {b }}$ \\ Universidad Católica de Murcia, Murcia, España. ${ }^{\mathrm{b}}$
}

\section{RESUMEN}

Introducción: La discapacidad social está presente en más del $50 \%$ de pacientes con esquizofrenia después de la remisión clínica. Hasta ahora no se han estudiado cómo difiere el procesamiento emocional entre los pacientes con alto o bajo funcionamiento social.

Método: Se hizo un estudio transversal, con 67 pacientes ambulatorios. Se utilizaron la Escala de Funcionamiento Social y el Test de Inteligencia Emocional.

Resultados: Se encontraron diferencias estadísticamente significativas en la Facilitación Emocional ( $\mathrm{p}=0.007$; $\mathrm{d}=$ 0.74), en el Manejo Emocional ( $\mathrm{p}=0.003$; $\mathrm{d}=0.83$ ) y en todas las dimensiones del funcionamiento social, con una diferencia de hasta 45.43 puntos entre los grupos.

Conclusiones: Los resultados contribuyen a la comprensión del papel que desempeña la facilitación y regulación emocional en el funcionamiento social.

\section{Palabras clave}

regulación emocional, facilitación emocional, esquizofrenia, funcionamiento social

\begin{abstract}
Introduction: Social disability is present in more than $50 \%$ of patients with schizophrenia after clinical remission. How emotional processing differs between patients with high and low social functioning has not been studied so far. Methods: A cross-sectional study was conducted, with 67 outpatients. The Social Functioning Scale and the Emotional Intelligence Test were used.

Results: Statistically significant differences were found in Emotional Facilitation $(\mathrm{p}=0.007$; $\mathrm{d}=0.74)$, in Emotional Management $(\mathrm{p}=0.003 ; \mathrm{d}=0.83)$ and in all dimensions of social functioning, with a difference of up to 45.43 points between the groups.

Conclusions: The results contribute to the understanding of the role played by emotional facilitation and regulation in social functioning.
\end{abstract}

\section{Keywords}

emotional regulation, emotional facilitation, squizophrenia, social functioning

\footnotetext{
${ }^{1}$ Correspondence about this article should be addresed to Dulce Romero-Ayuso: dulceromero@ugr.es

${ }^{2}$ Los autores declaran no tener conflicto de intereses en el presente estudio. Los autores agradecen a todos los usuarios por su participación en esta investigación.
} 


\section{EMOTIONAL REGULATION AND SOCIAL FUNCTIONING IN PATIENTS WITH SCHIZOPHRENIA}

La esquizofrenia es un trastorno mental grave, con una prevalencia de 4.6 por 1000 personas. Se estima que un uno por ciento de la población desarrollará alguna forma de esquizofrenia a lo largo de su vida (Barrios, Gómez-Benito, Pino, Rojo, \& Guilera, 2018). La esquizofrenia produce una gran desventaja social que causa una discapacidad social moderada y grave (Tandon, Nasrallah, \& Keshavan, 2009), llegando a ocupar el séptimo lugar en menores de 60 años (Menendez-Miranda et al., 2015). Parte de esta discapacidad se ha relacionado con la falta de habilidad en las interacciones sociales y los síntomas negativos de la enfermedad. Distintos estudios han mostrado que las personas con esquizofrenia presentan déficit en la cognición social, que incluye dificultad para procesar emociones, en la percepción social, así como para comprender predecir el comportamiento y las intenciones de otras personas, lo que se puede traducir en relaciones sociales pobres y reducidas (Barrios et al., 2018; Mancuso, Horan, Kern, \& Green, 2011). Estas dificultades se inician entre los 5 y 10 años después del inicio de la enfermedad (Olsson, Hjärthag, \& Helldin, 2016) y siguen presentes tras la remisión clínica en más de la mitad de los pacientes (Pinna, Deriu, et al., 2013; Pinna, Tusconi, et al., 2013). Incluso se ha indicado que las dificultades en la percepción emocional también están presentes en el síndrome de psicosis atenuada (Fusar-Poli et al., 2012) y en el trastorno esquizotípico de la personalidad (McClure, Harvey, Bowie, Iacoviello, \& Siever, 2013). Uno de los síntomas claves parece ser la experiencia emocional reducida, entendida como el retiro emocional, retiro social pasivo o evitación social activa, o abstinencia social (Al-Halabí et al., 2016; Robertson et al., 2014; Silberstein, Pinkham, Penn, \& Harvey, 2018). Las emociones juegan un rol fundamental en el funcionamiento social, facilitándonos información sobre el significado de las diferentes situaciones sociales y la respuesta emocional más adecuada (Kimhy et al., 2012). En el caso de las personas con esquizofrenia, la falta de conciencia o claridad emocional puede producir dificultades en la percepción emocional, incluido del afecto facial, y en consecuencia en la regulación emocional (Aghvinian \& Sergi, 2018) produciendo como consecuencia dificultades en la adaptación e integración social (Kimhy et al., 2012). Una conciencia emocional deficiente puede generar dificultades para identificar y describir sentimientos, con un menor número de contactos sociales (Kimhy et al., 2012). Por otro lado, una dificultad para regular las emociones afecta al control de nuestras emociones, cuándo y cómo las experimentamos o expresamos (Gross, Uusberg, \& Uusberg, 2019). En las personas con 
esquizofrenia se ha observado que utilizan en mayor medida la supresión emocional, relacionada con un funcionamiento social deficiente, frente a la reevaluación cognitiva, que permite modificar la forma en la que se piensa sobre un determinado acontecimiento con el fin de alterar su impacto emocional. La reevaluación cognitiva está más relacionada con una emoción positiva y sentimientos generales de bienestar (Kimhy et al., 2012). Estas dificultades son persistentes en las distintas fases de la enfermedad y a lo largo del tiempo (Frajo-Apor, Pardeller, Kemmler, Welte, \& Hofer, 2016; Kee et al., 2009).

La investigación en esquizofrenia se ha centrado en su mayoría sobre la capacidad de detectar información emocional de las expresiones faciales de los otros (Kee et al., 2009). Recientemente, algunos estudios han intentado conocer la relación entre las estrategias de regulación emocional y su repercusión social en pacientes con esquizofrenia (Moran \& Kring, 2018). De este modo, se ha relacionado una menor supresión y una mayor reevaluación con un mejor funcionamiento social en la esquizofrenia (Kimhy et al., 2012). Además, el uso habitual de la supresión se ha relacionado con un funcionamiento social más pobre, mientras que la reevaluación se relacionó con la disminución de los niveles de depresión y síntomas negativos (Perry, Henry, \& Grisham, 2011).

En la esquizofrenia el deterioro del funcionamiento social se considera un aspecto crucial y un elemento básico de la sintomatología, como queda de manifiesto con su inclusión entre los criterios diagnósticos que definen este trastorno psiquiátrico (Tandon et al., 2009). Estas dificultades pueden afectar al funcionamiento social en distintas actividades de la vida diaria, tales como las actividades básicas, de autocuidado (higiene, vestuario y alimentación), rendimiento ocupacional (trabajo remunerado, estudios, labores domésticas), funcionamiento en roles familiares y en roles sociales (participación con otros miembros de la comunidad en actividades de esparcimiento y de otro tipo) (Pinna, Deriu, et al., 2013). Además, un bajo funcionamiento social se ha relacionado con una mayor sobrecarga en el cuidador, que afecta a la carga económica, y la falta de tiempo libre del mismo (Pinna, Tusconi, et al., 2013).

A pesar de que hay estudios sobre la importancia y la persistencia de los déficit en el funcionamiento social en pacientes con esquizofrenia y su relación con la inteligencia emocional, para el conocimiento de los autores no hay ningún estudio que haya estudiado las diferencias en la inteligencia emocional en pacientes con esquizofrenia con alto y bajo funcionamiento social. Dada la relevancia de la disfunción social en personas con esquizofrenia y su relación con la conciencia 
y regulación emocional, nuestros objetivos son: 1) ampliar los hallazgos de los estudios previos en los que se ha constatado diferencias en estas variables al comparar pacientes con esquizofrenia y controles sanos; y 2) conocer la relación entre funcionamiento social alto o bajo con la percepción emocional, facilitación y regulación emocional.

\section{Método}

El diseño corresponde a un estudio transversal, en el que se recogen diferentes variables en un mismo punto en el tiempo.

\section{Participantes}

Los participantes debían cumplir los siguientes criterios de inclusión: 1) Personas con trastorno mental grave, con el diagnóstico de Esquizofrenia (F20) según los criterios del CIE-10, que presentan dificultades significativas en el funcionamiento psicosocial y para el desarrollo de las actividades básicas de autocuidados; 2) Adultos tanto hombres como mujeres de edad comprendida entre 20 y 55 años; 3) Estar estable psicopatológicamente; 4) No tener trastornos graves de comportamiento; 5) No consumir alcohol u otros tóxicos en la actualidad; 6) Ser usuarios de la Unidad de Rehabilitación (UR) y no institucionalizados; 7) Tener un funcionamiento social alto, según la Escala de Funcionamiento Social (SFS), (>106 en el SFS) o bajo (<96 en el SFS); y 8) Dar su consentimiento para participar. Los criterios de exclusión de la muestra fueron los siguientes: 1) Discapacidad intelectual y demencia; 2) Gran invalidez; 3) Evidencias de rasgos antisociales; 4) No consentimiento por parte del paciente o familia.

De los 74 pacientes que inicialmente mostraron interés en participar sólo 67 cumplieron todos los criterios de inclusión.

\section{Procedimiento}

Los sujetos de este estudio participaron voluntariamente, siendo la población de referencia mujeres y hombres con trastorno mental grave de las áreas de Murcia pertenecientes a las zonas de Caravaca de la Cruz, Cartagena, Lorca y Murcia centro, que acuden a las Unidades de Rehabilitación (UR). El tipo de muestreo fue intencional, no probabilístico. La evaluación se realizó en una sesión, de 1 hora. Después de aceptar su participación, se procedió a programar la 
primera sesión de forma individualizada, ajustándose a la disponibilidad del sujeto y de los investigadores. Cada participante fue informado de las características del estudio y del cumplimiento de la legislación relativa a la protección de datos. Todos los participantes dieron su consentimiento informado por escrito.

El estudio fue aprobado por el Comité de Ética e Investigación Clínica del Hospital Virgen de la Arrixaca.

\section{Instrumentos}

Todos los participantes fueron evaluados con la Escala de Funcionamiento Social (SFS) (Mancuso et al., 2011) y con el Test de Inteligencia Emocional de Mayer-Salovey-Caruso (MSCEIT) (Mayer, Salovey, \& Caruso, 2011; Mayer et al., 2011).

La Escala de Funcionamiento Social (SFS) mide distintos aspectos de la vida social, se caracteriza por estar menos influenciada por las características demográficas y clínicas que otras escalas y añade información valiosa como un suplemento a las herramientas de evaluación en salud mental (Menendez-Miranda et al., 2015). Está diseñada para su uso en programas de intervención familiar con pacientes esquizofrénicos, intentando cubrir el vacío existente en la evaluación del funcionamiento social y proporcionar una evaluación detallada de las fortalezas y debilidades relativas del individuo y guiarlos para adaptar las intervenciones individualizadas (Birchwood, Smith, Cochrane, Wetton, \& Copestake, 1990). Evalúa los aspectos que se juzgan fundamentales a la hora de mantener al paciente en la comunidad. Se incluyen siete áreas: 1) Aislamiento/Integración social; 2) Comunicación interpersonal; 3) Independencia-Ejecución; 4) Independencia-Competencia; 5) Tiempo Libre; 6) Actividades Prosociales; y 7) Empleo/Ocupación. Esta escala permite diferenciar entre la falta de competencia para realizar una tarea para una vida independiente y la realización incorrecta. Hay dos versiones: una heteroinformada, que puede ser contestada por los familiares, y una versión autoinformada, que cumplimentada por el propio paciente. La escala SFS está validada al castellano (Olivares-Díez \& Torres, 2005). La consistencia interna para la escala global es de 0.80 , oscilando entre 0.69 y 0.87 para las diferentes subescalas. También muestra buenas propiedades psicométricas respecto a la fiabilidad inter-jueces para la escala total (0.94) y para las subescalas, oscilando entre 0.69 y 0.96. Asimismo, muestra valores satisfactorios de validez concurrente cuando se compara con medidas que evalúan constructos similares. Además, la SFS permite diferenciar entre pacientes con un alto, 
medio y bajo funcionamiento social. Puntuaciones inferiores a 96 puntos indican un bajo funcionamiento social, entre 96 y 106 un funcionamiento social medio y puntuaciones superiores a 106 funcionamiento social alto (Olivares-Díez \& Torres, 2005).

El Test de Inteligencia Emocional de Mayer-Salovey-Caruso (MSCEIT) es un instrumento que permite evaluar el nivel general de inteligencia emocional. También se puede utilizar desde el punto de vista clínico para mejorar el conocimiento de sí mismo y determinar en qué áreas puede ser aconsejable desarrollar su conocimiento emocional (Trinidad \& Johnson, 2002). Se puede utilizar a partir de los 17 años. Su cumplimentación dura aproximadamente entre 30 y 45 minutos. La versión española del MSCEIT versión 2.0 tiene una fiabilidad global de 0.95, con una fiabilidad de 0.93 para el área experiencial y de 0.90 para el área estratégica. Además, se han obtenido elevadas correlaciones entre las puntuaciones obtenidas con los sistemas de corrección español y el original $(r=0.99)$. Los análisis factoriales replican la estructura original del instrumento, compuesto por: una puntuación total del cociente de inteligencia emocional (CIE); dos áreas, que son el cociente de inteligencia emocional experiencial (CIEX) y el cociente de inteligencia emocional estratégica (CIES); y cuatro habilidades básicas: 1) percepción emocional (CIEP); 2) facilitación emocional (CIEF); 3) comprensión emocional (CIEC) y 4) manejo emocional (CIEM); el test está formado por ocho tareas: caras, paisajes y diseños, sensaciones, facilitación, combinaciones, cambios, regulación de emociones y relaciones emocionales. Este instrumento ha sido considerado útil para ser empleado con muestras clínicas para detectar aquellos individuos con baja inteligencia emocional. Las puntuaciones más altas en el MSCEIT corresponden a niveles más altos de funcionamiento adaptativo (Kee et al., 2009). Además, se ha indicado que es una buena medida para evaluar la inteligencia emocional en población con esquizofrenia (Kee et al., 2009). Existen varios estudios que han analizado los factores del MSCEIT con población con esquizofrenia, encontrando en ambos casos dos factores principales: regulación emocional y percepción emocional. Además, en algunos países, la sección de regulación emocional se ha incorporado al protocolo de evaluación rutinaria de pacientes con esquizofrenia (Eack, PogueGeile, Greeno, \& Keshavan, 2009).

\section{Análisis estadístico}

Los análisis estadísticos mencionados se realizaron utilizando IBM SPSS Statistics para Windows (versión 26.0, IBM Corp., Armonk, NY). Se consideró un nivel de significación 
estadística con un valor $p<0.05$ (bilateral). Las características de los participantes se analizaron utilizando estadística descriptiva simple. Se realizó un contraste de hipótesis a través de la prueba $t$ de student para muestras independientes y se estudio la correlación entre las variables de funcionamiento social y las dimensiones de percepción, comprensión, facilitación y regulación emocional del MSCEIT.

\section{Resultados}

En el análisis de variables sociodemográficas se observa que de los 67 pacientes con una media \pm desviación estándar de edad de $39.19 \pm 1.02$ años, 14 (20.85\%) eran mujeres. El 74.6\% cumplían los criterios diagnósticos de esquizofrenia tipo desorganizada. El 17.9\% no tenía estudios, el $37.3 \%$ tenía cursados los estudios obligatorios, 38\% había cursado bachillerato o formación profesional y sólo el 6\% tenía estudios universitarios. El 100\% residía en una vivienda familiar. El $80.6 \%$ convivía con su padres o familiares, el $16.4 \%$ vivía solo y el 3\% lo hacía en pareja.

Respecto al tratamiento farmacológico, el 14.9\% estaba sólo con tratamiento antipsicótico y el $85.1 \%$ tenía más de un tratamiento farmacológico a la vez. El 35.82\% quedó clasificado como bajo funcionamiento social. La edad media \pm desviación estándar de edad en el grupo con bajo funcionamiento social fue de $39.79 \pm 8.5$ y para el grupo con alto funcionamiento social fue de $38.86 \pm 8.4$ ( $p=0.668)$. Las características sociodemográficas y clínicas de la muestra se recogen en la Tabla 1. No hubo diferencias estadísticamente significativas en ninguna variable sociodemográfica ni clínica.

En relación a la comparación entre los dos grupos, alto y bajo funcionamiento social, los resultados muestran diferencias estadísticamente significativas en todas las dimensiones del funcionamiento social: Aislamiento e integración social $(p<0.001 ; d=1.48)$; comunicación interpersonal $(p<0.001 ; d=1.17)$; Independencia ejecución $(p<0.001 ; d=2.18)$; Independenciacompetencia ( $p<0.001 ; d=0.91)$; Tiempo Libre $(p<0.001 ; d=1.60)$; Actividades Sociales $(p<0.001$; $d=0.76$ ); Empleo/ocupación ( $p=0.003 ; d=0.76$ ). Tomadas en conjunto todas las puntuaciones, se observa un tamaño del efecto mayor para el funcionamiento social total ( $p<0.001 d=3.25$ ), con una diferencia de hasta 45.43 puntos entre el grupo de alto y bajo funcionamiento social.

Respecto a las diferencias encontradas entre el grupo con alto y bajo funcionamiento social y la inteligencia emocional, los resultados mostraron diferencias en la Facilitación Emocional 
(CIEF) $(p=0.007 ; d=0.74)$ y en el Manejo Emocional (CIEM) $(p=0.003 ; d=0.83)$. En el resto de variables no es encontraron diferencias estadísticamente significativas.

Tabla 1.

Características sociodemográficas y clínicas de la muestra según el nivel de funcionamiento social del SFS.

\begin{tabular}{|c|c|c|c|c|c|c|}
\hline & & $\mathbf{n}$ & $\%$ & $\mathbf{n}$ & $\%$ & $p$ \\
\hline \multirow[t]{2}{*}{ Sexo } & Hombre & 17 & 70.8 & 36 & 83.7 & .213 \\
\hline & Mujer & 7 & 29.2 & 7 & 16.3 & \\
\hline \multirow[t]{2}{*}{ Grupos Diagnósticos } & Esquizofrenia tipo desorganizado & 17 & 70.8 & 33 & 76.7 & .594 \\
\hline & Otros tipos de esquizofrenia & 7 & 29.2 & 10 & 23.3 & \\
\hline \multirow{5}{*}{ Tratamiento farmacológico } & Sin tratamiento & 0 & 0 & 0 & 0 & .065 \\
\hline & Tratamiento antipsicótico & 1 & 4.2 & 9 & 20.9 & \\
\hline & Tratamientos ansiolíticos & 0 & 0 & 0 & 0 & \\
\hline & Otro tratamiento & 0 & 0 & 0 & 0 & \\
\hline & $\begin{array}{l}\text { Más de un tratamiento } \\
\text { farmacológico }\end{array}$ & 23 & 95.8 & 34 & 79.1 & \\
\hline \multirow[t]{6}{*}{ Nivel de estudios } & Sin estudios & 4 & 16.7 & 8 & 18.6 & .229 \\
\hline & Primaria & 9 & 37.5 & 13 & 30.2 & \\
\hline & Secundaria & 0 & 0 & 3 & 7 & \\
\hline & Bachillerato o FP1 & 10 & 41,7 & 10 & 23.3 & \\
\hline & FP2 o modulo profesional & 1 & 4,2 & 5 & 11.6 & \\
\hline & Universitarios & 0 & 0 & 4 & 9.3 & \\
\hline \multirow[t]{3}{*}{ Tipo de vivienda } & Vivienda familiar & 24 & 100 & 43 & 100 & - \\
\hline & Vivienda protegida & 0 & 0 & 0 & 0 & \\
\hline & Vivienda supervisada & 0 & 0 & 0 & 0 & \\
\hline \multirow[t]{3}{*}{ Convivencia } & Con padres o familiares & 21 & 87.5 & 33 & 76.6 & .430 \\
\hline & Solo & 3 & 12.5 & 8 & 18.5 & \\
\hline & Con pareja & 0 & 0 & 2 & 4.6 & \\
\hline
\end{tabular}

Finalmente, el estudio de la correlación entre las cuatro dimensiones de la inteligencia emocional evaluadas y la dimensiones del SFS mostró que la Independencia- como competencia correlacionaba con todas las dimensiones: percepción emocional $(\mathrm{r}=0.285 ; p=0.019)$, facilitación emocional $(\mathrm{r}=0.303 ; p=0.013)$, comprensión emocional $(\mathrm{r}=0.368 ; p=0.002)$ y con manejo emocional $(\mathrm{r}=0.247 ; p=0.044)$. En cambio, la dimensión de Independencia, como desempeño o 
ejecución, sólo mostró correlación significativa con la facilitación emocional $(\mathrm{r}=0.309 ; p=0.011)$ y regulación emocional $(\mathrm{r}=0.342 ; p=0.005)$. Por último, la dimensión de interacción- aislamiento social sólo mostró una correlación positiva significativa con la regulación emocional $(\mathrm{r}=0.243$; $p=0.048)$.

Tabla 2

Inteligencia Emocional según el funcionamiento social alto y bajo en pacientes con esquizofrenia

\begin{tabular}{|c|c|c|c|c|c|c|c|c|c|c|}
\hline & \multicolumn{2}{|c|}{$\begin{array}{c}\text { Grupo con Alto } \\
\text { Funcionamiento } \\
\text { Social } \\
\end{array}$} & \multicolumn{2}{|c|}{$\begin{array}{c}\text { Grupo con Bajo } \\
\text { Funcionamiento } \\
\text { Social } \\
\end{array}$} & \multirow[b]{2}{*}{$\begin{array}{l}\text { Diferencia } \\
\text { de Medias }\end{array}$} & \multirow[b]{2}{*}{$\mathbf{t}$} & \multirow[b]{2}{*}{$p$} & \multirow[b]{2}{*}{$\begin{array}{c}d \\
\text { Cohen }\end{array}$} & \multirow[b]{2}{*}{$\begin{array}{c}\text { Límite } \\
\text { inferior }\end{array}$} & \multirow[b]{2}{*}{$\begin{array}{c}\text { Límite } \\
\text { superior }\end{array}$} \\
\hline & Media & SD & Media & $S D$ & & & & & & \\
\hline Aislamiento Social & 11.07 & 1.86 & 8.42 & 1.64 & -2.65 & -5.81 & $<0.001$ & -1.48 & -3.36 & -1.74 \\
\hline Comunicación interpersonal & 6.86 & 1.92 & 4.25 & 2.47 & -2.61 & -4.80 & $<0.001$ & -1.17 & -3.69 & -1.52 \\
\hline Autonomía-Ejecución & 26.90 & 5.84 & 15 & 5.02 & -11.90 & -8.39 & $<0.001$ & -2.18 & -14.73 & -9.07 \\
\hline Autonomía-Competencia & 35.69 & 2.81 & 30.5 & 7.49 & -5.19 & -3.27 & $<0.001$ & -0.91 & -7.74 & -2.65 \\
\hline Ocio & 20.27 & 6.20 & 11.62 & 4.41 & -8.65 & -6.02 & $<0.001$ & -1.60 & -11.52 & -5.78 \\
\hline Actividades Prosociales & 22.16 & 9.99 & 9 & 4.76 & -13.16 & -6.06 & $<0.001$ & -1.68 & -17.49 & -8.82 \\
\hline Empleo-Ocupación & 2.95 & 1.57 & 1.70 & 1.68 & -1.24 & -3.03 & $<0.003$ & $-0,76$ & -2.06 & -.424 \\
\hline $\begin{array}{l}\text { Cociente de Inteligencia } \\
\text { Emocional Experiencial (CIEX) }\end{array}$ & 96.39 & 12.66 & 92.58 & 11.90 & -3.81 & -3.81 & .232 & -0.30 & -10.12 & 2.49 \\
\hline $\begin{array}{l}\text { Cociente de Inteligencia } \\
\text { Emocional Estratégico (CIES) }\end{array}$ & 90.09 & 10.42 & 85.25 & 9.81 & -4.84 & -4.84 & 0.067 & -0.47 & -10.03 & .355 \\
\hline Percepción emocional (CIEP) & 97.11 & 15.21 & 96.66 & 15.21 & -.449 & -.449 & 0.904 & -0.03 & -9.97 & .293 \\
\hline Facilitación emocional (CIEF) & 96.86 & 10.76 & 89.83 & 8.03 & -7.02 & $-7,02$ & 0.007 & -0.74 & -12.05 & -1.99 \\
\hline Compresión emocional (CIEC) & 86.11 & 9.60 & 84.75 & 11.37 & -1.36 & -1.36 & 0.603 & -0.12 & -6.59 & 3.85 \\
\hline Manejo emocional (CIEM) & 98.53 & 13.84 & 88.54 & 9.89 & -9.99 & -9.99 & 0.003 & -0.83 & -15.83 & -4.14 \\
\hline CIE Total & 92.34 & 11.21 & 87.62 & 10.11 & -4.72 & -4.72 & 0.092 & -0.44 & -10.23 & .656 \\
\hline SFS Total & 125.93 & 16.71 & 80.50 & 10.49 & -45.43 & -45.43 & $<0.001$ & -3.25 & -52.97 & -37.89 \\
\hline
\end{tabular}

\section{Discusión}

El objetivo de este trabajo era conocer las diferencias entre los pacientes con alto y bajo funcionamiento social y su relación con las destrezas emocionales afectadas en los usuarios ambulatorios de los recursos mentales comunitarios. En general, más de la mitad de la muestra $(64.18 \%)$ presentaba un alto funcionamiento social. En el grupo con bajo funcionamiento social, se han encontrado más dificultades en la realización de actividades prosociales, con 13.16 puntos de diferencia, en la ejecución de actividades de autonomía personal, con una diferencia de hasta 
11.90 puntos entre ambos grupos, así como en la participación en actividades de ocio y gestión del tiempo libre, con 8.65 puntos de diferencia.

Los resultados de este estudio muestran que las destrezas emocionales más deficitarias en pacientes con esquizofrenia con bajo funcionamiento social, que acuden a las unidades de rehabilitación, corresponden a la regulación reflexiva de la emoción y la facilitación emocional. La facilitación emocional puede ser entendida como la habilidad para reconducir y priorizar el pensamiento basado en los sentimientos asociados a objetos, situaciones y personas, permitiendo solucionar problemas y tomar decisiones. Supone la habilidad para tener en cuenta los sentimientos cuando razonamos o solucionamos problemas, nos permite priorizar nuestros procesos cognitivos básicos, ayudándonos a centrar nuestra atención en lo que es realmente importante (Frajo-Apor et al., 2016). Por otro lado, la regulación, es una habilidad más compleja, que incluye las siguientes destrezas: 1) habilidad para estar abierto tanto a los estados emocionales positivos como negativos; 2) habilidad para reflexionar sobre las emociones y determinar la utilidad de su información; 3) habilidad para monitorizar reflexivamente nuestras emociones y las de los otros, así como reconocer su influencia; y 4) habilidad para regular nuestras emociones y la de los demás sin minimizarlas ni exagerarlas. Por tanto, se refiere a la habilidad para controlar y mostrar emociones acordes a cada situación, ser capaz de iniciar, mantener un terminado estado emocional, en función de las propias emociones y las de los demás. Permite controlar las emociones negativas y centrarse en las placenteras (Mayer, Salovey, Caruso, \& Cherkasskiy, 2011; Mayer, Salovey, \& Caruso, 2008).

Nuestros resultados son consistente con los estudios previos que muestran peor rendimiento en pacientes con esquizofrenia al compararlos con grupo de adultos sanos, en tareas de manejo o regulación emocional, con peor rendimiento desempeño en las pruebas de identificar, entender y manejar las emociones (Frajo-Apor et al., 2016; Kee et al., 2009) y con puntuaciones significativamente más bajas en funcionamiento social (Dawson, Kettler, Burton, \& Galletly, 2012; Kee et al., 2009). Asimismo, nuestros resultados son consistentes con los estudios que han mostrado que los déficit en el reconocimiento de emociones como un elemento clave en la esquizofrenia, relacionándose con la gravedad de los síntomas, un funcionamiento social reducido y consecuentemente con dificultades en la integración social (Lado-Codesido, Méndez Pérez, Mateos, Olivares, \& García Caballero, 2019). Los resultados están en consonancia con otros estudios que han mostrado que los pacientes con esquizofrenia presentan una disminución de su 
actividad social, que podrían ser consecuencia de los déficit en cognición social, dentro de la cual está incluido el proceso de comprensión emocional que requiere la interpretación de la expresión mímica, de la entonación y prosodia afectiva del lenguaje, comprender la intencionalidad del otro en la interacción, las expresiones faciales ambivalentes y no prototípicas (Wölwer et al., 2008).

Los resultados de este estudio añaden que estás diferencias se producen fundamentalmente en los pacientes con bajo funcionamiento social, versus los pacientes con alto funcionamiento social en los que se observan diferencias en la facilitación emocional y especialmente en la regulación emocional. Estos resultados podrían relacionarse con lo observado en otros estudios que indican que en los pacientes con esquizofrenia que utilizan como estrategia de regulación emocional la supresión, muestran una mayor emoción negativa, una reducción de la emoción positiva y una disminución de la interacción social en su vida diaria (Moran \& Kring, 2018).

Por otro lado, otros estudios han encontrado que en los pacientes con esquizofrenia y con ansiedad social, que es muy prevalente en esta población, llegando a un 30\%, tienen más dificultades a la hora de reconocer caras emocionales neutras, muestran peor funcionamiento social, tanto en la comunicación interpersonal como en el compromiso en las conversaciones y muestran una menor autoestima (Lecomte, Théroux, Paquin, Potvin, \& Achim, 2019).

Los pacientes con mayor puntuación en la SFS mostraron una mayor puntuación en el MSCEIT, similar a lo que han indicado estudios previos (Kee et al., 2009), relacionándose con niveles de funcionamiento adaptativo más alto en las relaciones con padres, amigos, de pareja (Lopes, Salovey, Coté, \& Beers, 2005) y de éxito a nivel laboral o académico (Lopes, Grewal, Kadis, Gall, \& Salovey, 2006). La identificación de emociones correlacionó significativamente con el ajuste psicosocial y el rol en la comunidad, sugiriendo que los pacientes con esquizofrenia con menor puntuación en el MSCEIT muestran menor ajuste psicosocial y menor competencia en la comunidad (Kee et al., 2009).

Respecto a la relación entre las estrategias de regulación emocional con el funcionamiento social, se ha indicado que un mayor uso de la supresión se asocia con un funcionamiento social más deficiente, con menor calidad de las amistades, menor sensibilidad interpersonal, menos actividades prosociales y más conflicto sociales en los adultos jóvenes (Kimhy et al., 2012; Lopes et al., 2005).

Es interesante señalar que no encontramos diferencias entre los pacientes con alto y bajo funcionamiento social en la percepción y comprensión emocional. Sin embargo, otros autores han 
señalado que en pacientes con esquizofrenia al compararlos con población sana, se produce un déficit en la comprensión emocional. Nuestros hallazgos podrían ser debidos a que en ambos grupos está afectada por igual la comprensión emocional y lo que realmente los diferencia en el grado de bajo o alto funcionamiento son los procesos más complejos de facilitación y regulación emocional. Todo ello sugiere que, estas dos habilidades pueden explicar la gravedad de la discapacidad social y la importancia de su intervención de manera más precisa e intensiva en pacientes con bajo funcionamiento social. Los resultados de nuestro estudio son relevantes de cara a los programas de recuperación /rehabilitación. Ayudar a los pacientes a regular las respuestas emocionales en experiencias emocionales menos negativas, podría aumentar su funcionamiento social. Las emociones negativas pueden desencadenar síntomas psicóticos, lo que hace aún más relevante que aprendan estrategias de regulación efectivas (Grezellschak, Lincoln, \& Westermann, 2015). Además, se ha constatado que las personas con esquizofrenia son capaces de modular su regulación emocional siguiendo instrucciones explícitas (Moran \& Kring, 2018). El desarrollo de las habilidades emocionales en los pacientes ambulatorios puede ser crucial para el desarrollo de estrategias efectivas de prevención y tratamiento, ya que se ha observado que el desarrollo de la empatía y habilidades de comprensión emocional es un buen predictor del éxito del tratamiento (Rus-Calafell, Gutiérrez-Maldonado, Ortega-Bravo, Ribas-Sabaté, \& CaqueoUrízar, 2013).

Nuestro estudio tiene varias limitaciones. Primero, la selección de los pacientes se realizó utilizando un muestreo de conveniencia no probabilística. Esto puede limitar la extrapolación de los resultados, aunque se ha demostrado la utilidad de este método en estudios exploratorios como este (Hernández, Fernández, \& Baptista, 2010). Otra limitación es el reducido número de participantes. Por lo tanto, sería aconsejable diseñar nuevos estudios que incluyan un mayor número de participantes. Finalmente, otra limitación, podría ser que a la hora de cumplimentar el SFS se hubiera producido un sesgo de deseabilidad social por parecer más autónomo. El uso del autoinforme aún puede haber influido en los resultados. Aunque la metodología empleada ha sido la habitual y con la que se ha validado el instrumento en distintas culturas.

Estudios futuros podrían estudiar las habilidades emocionales mediante tareas de laboratorio y correlatos psicofisiológicos en pacientes con alto y bajo funcionamiento social. También sería de interés utilizar pruebas de rendimiento con validez ecológica, como pueden ser 
las tareas virtuales, para conocer el funcionamiento social y ver su relación con los correlatos psicofisiológicos.

En conclusión y en vista de lo anterior, los resultados sugieren que los pacientes con esquizofrenia con bajo funcionamiento social muestran dificultades para el uso de emociones de forma adaptativa. Nuestros hallazgos están de acuerdo con la extensa literatura sobre ciencia afectiva básica que apunta a fuertes vínculos entre bajo funcionamiento social y déficits en la facilitación y regulación emocional (Kimhy et al., 2012). 


\section{Referencias}

Aghvinian, M., \& Sergi, M. J. (2018). Social functioning impairments in schizotypy when social cognition and neurocognition are not impaired. Schizophrenia Research Cognition, 14, 713. https://doi.org/10.1016/j.scog.2018.07.001

Al-Halabí, S., Sáiz, P. A., Garrido, M., Galván, G., Casares, M. J., Bobes-Bascarán, M. T., Bobes, J. (2016). Psychometric properties of a Spanish-version of the Schizophrenia Objective Functioning Instrument (Sp-SOFI). International Journal of Clinical and Health Psychology, 16(1), 58-75. https://doi.org/10.1016/j.ijchp.2015.07.004

Barrios, M., Gómez-Benito, J., Pino, O., Rojo, E., \& Guilera, G. (2018). Functioning in patients with schizophrenia: A multicentre study evaluating the clinical perspective. Psychiatry Research, 270, 1092-1098. https://doi.org/10.1016/j.psychres.2018.05.079

Birchwood, M., Smith, J., Cochrane, R., Wetton, S., \& Copestake, S. (1990). The Social Functioning Scale. The development and validation of a new scale of social adjustment for use in family intervention programmes with schizophrenic patients. The British Journal of Psychiatry: the journal of mental science, 157, 853-859. https://doi.org/10.1192/bjp.157.6.853

Dawson, S., Kettler, L., Burton, C., \& Galletly, C. (2012). Do people with schizophrenia lack emotional intelligence? Schizophrenia Research and Treatment, 2012, 495174. https://doi.org/10.1155/2012/495174

Eack, S. M., Pogue-Geile, M. F., Greeno, C. G., \& Keshavan, M. S. (2009). Evidence of factorial variance of the Mayer-Salovey-Caruso Emotional Intelligence Test across schizophrenia and normative samples. Schizophrenia Researh, 114(1-3), 105-109. https://doi.org/10.1016/j.schres.2009.05.011

Frajo-Apor, B., Pardeller, S., Kemmler, G., Welte, A. S., \& Hofer, A. (2016). Emotional Intelligence deficits in schizophrenia: The impact of non-social cognition. Schizophrenia Research, 172(1-3), 131-136. https://doi.org/10.1016/j.schres.2016.02.027

Fusar-Poli, P., Bonoldi, I., Yung, A. R., Borgwardt, S., Kempton, M. J., Valmaggia, L., Barale, F., Caverzasi, E., McGuire, P. (2012). Predicting psychosis: meta-analysis of transition outcomes in individuals at high clinical risk. Archives of General Psychiatry, 69(3), 220229. https://doi.org/10.1001/archgenpsychiatry.2011.1472

Grezellschak, S., Lincoln, T. M., \& Westermann, S. (2015). Cognitive emotion regulation in patients with schizophrenia: Evidence for effective reappraisal and distraction. Psychiatry Research, 229(1-2), 434-439. https://doi.org/10.1016/j.psychres.2015.05.103

Gross, J. J., Uusberg, H., \& Uusberg, A. (2019). Mental illness and well-being: an affect regulation perspective. World Psychiatry, 18(2), 130-139. https://doi.org/10.1002/wps.20618

Hernández, R., Fernández, C., \& Baptista, M. P. (2010). Metodología de la Investigación (Quinta Edición ed.). México, D.F.: Mc-Graw Hill.

Kee, K. S., Horan, W. P., Salovey, P., Kern, R. S., Sergi, M. J., Fiske, A. P., Lee,J., Subotnik, K.L., Nuechterlein, K., Sugar, C.A, \& Green, M. F. (2009). Emotional intelligence in schizophrenia. Schizophrenia Research, 107(1), 61-68. https://doi.org/10.1016/j.schres.2008.08.016

Kimhy, D., Vakhrusheva, J., Jobson-Ahmed, L., Tarrier, N., Malaspina, D., \& Gross, J. J. (2012). Emotion awareness and regulation in individuals with schizophrenia: Implications for social functioning. Psychiatry Research, 200(2-3), 193-201. https://doi.org/10.1016/j.psychres.2012.05.029 
Lado-Codesido, M., Méndez Pérez, C., Mateos, R., Olivares, J. M., \& García Caballero, A. (2019). Improving emotion recognition in schizophrenia with "VOICES": An on-line prosodic self-training. PLoS One, 14(1), e0210816. https://doi.org/10.1371/journal.pone.0210816

Lecomte, T., Théroux, L., Paquin, K., Potvin, S., \& Achim, A. (2019). Can Social Anxiety Impact Facial Emotion Recognition in Schizophrenia? Journal of Nervous and Mental Disease, 207(3), 140-144. https://doi.org/10.1097/NMD.0000000000000934

Lopes, P. N., Grewal, D., Kadis, J., Gall, M., \& Salovey, P. (2006). Evidence that emotional intelligence is related to job performance and affect and attitudes at work. Psicothema, 18 Suppl, 132-138.

Lopes, P. N., Salovey, P., Coté, S., \& Beers, M. (2005). Emotion regulation abilities and the quality of social interaction. Emotion, 5(1), 113-118. https://doi.org/10.1037/1528-3542.5.1.113

Mancuso, F., Horan, W. P., Kern, R. S., \& Green, M. F. (2011). Social cognition in psychosis: multidimensional structure, clinical correlates, and relationship with functional outcome. Schizophrenia Research, 125(2-3), 143-151. https://doi.org/10.1016/j.schres.2010.11.007

Mayer, J., Salovey, P., Caruso, D., \& Cherkasskiy, L. (2011). Emotional intelligence. In R. J. S. J. Kaufman (Ed.), The Cambridge handbook of intelligence (3rd ed.). New York: Cambridge University Press.

Mayer, J. D., Salovey, P., \& Caruso, D. R. (2008). Emotional intelligence: new ability or eclectic traits? American Psychologist, 63(6), 503-517. https://doi.org/10.1037/0003$\underline{066 X .63 .6 .503}$

Mayer, J. D., Salovey, P., \& Caruso, D. R. (2011). Test de Inteligencia Emocional Mayer-SaloveyCaruso. Madrid: Tea Ediciones.

McClure, M. M., Harvey, P. D., Bowie, C. R., Iacoviello, B., \& Siever, L. J. (2013). Functional outcomes, functional capacity, and cognitive impairment in schizotypal personality disorder. Schizophrenia Research, 144(1-3), 146-150. https://doi.org/10.1016/j.schres.2012.12.012

Menendez-Miranda, I., Garcia-Portilla, M. P., Garcia-Alvarez, L., Arrojo, M., Sanchez, P., Sarramea, F., Gomar, J., Bobes-Bascaran, M.T., Sierra, P., Saiz, P.A. \& Bobes, J. (2015). Predictive factors of functional capacity and real-world functioning in patients with $\begin{array}{llll}\text { schizophrenia. } \quad \text { European } & \text { 30(5), }\end{array}$ https://doi.org/10.1016/j.eurpsy.2014.12.011

Moran, E. K., \& Kring, A. M. (2018). Anticipatory Emotion in Schizophrenia. Clinical Psychological Science: a journal of the Association Psychological Science, 6(1), 63-75. https://doi.org/10.1177/2167702617730877

Olivares-Díez, J., \& Torres, A. (2005). Validación en castellano de la Social Functioning Scale (Escala de Funcionamiento Social). Actas Españolas de Psiquiatría, 33(4), 216-220.

Olsson, A. K., Hjärthag, F., \& Helldin, L. (2016). Predicting real-world functional milestones in schizophrenia. Psychiatry Research, 242, https://doi.org/10.1016/j.psychres.2016.05.015

Perry, Y., Henry, J. D., \& Grisham, J. R. (2011). The habitual use of emotion regulation strategies in schizophrenia. The British Journal of Clinical Psychology, 50(2), 217-222. https://doi.org/10.1111/j.2044-8260.2010.02001.x

Pinna, F., Deriu, L., Lepori, T., Maccioni, R., Milia, P., Sarritzu, E., Tusconi, M., Carpiniello, B., \& Cagliary Recovery Study Group. (2013). Is it true remission? A study of remitted patients affected by schizophrenia and schizoaffective disorders. Psychiatry Research, 210(3), 739-744. https://doi.org/10.1016/j.psychres.2013.08.022 
Pinna, F., Tusconi, M., Bosia, M., Cavallaro, R., Carpiniello, B., \& Study, C. R. G. (2013). Criteria for symptom remission revisited: a study of patients affected by schizophrenia and schizoaffective disorders. BMC Psychiatry, 13, 235. https://doi.org/10.1186/1471-244X13-235

Robertson, B. R., Prestia, D., Twamley, E. W., Patterson, T. L., Bowie, C. R., \& Harvey, P. D. (2014). Social competence versus negative symptoms as predictors of real world social functioning in schizophrenia. Schizophrenia Research, 160(1-3), 136-141. https://doi.org/10.1016/j.schres.2014.10.037

Rus-Calafell, M., Gutiérrez-Maldonado, J., Ortega-Bravo, M., Ribas-Sabaté, J., \& Caqueo-Urízar, A. (2013). A brief cognitive-behavioural social skills training for stabilised outpatients with schizophrenia: a preliminary study. Schizophrenia Research, 143(2-3), 327-336. https://doi.org/10.1016/j.schres.2012.11.014

Silberstein, J. M., Pinkham, A. E., Penn, D. L., \& Harvey, P. D. (2018). Self-assessment of social cognitive ability in schizophrenia: Association with social cognitive test performance, informant assessments of social cognitive ability, and everyday outcomes. Schizophrenia Research, 199, 75-82. https://doi.org/10.1016/j.schres.2018.04.015

Tandon, R., Nasrallah, H. A., \& Keshavan, M. S. (2009). Schizophrenia, "just the facts" 4. Clinical features and conceptualization. Schizophrenia Research, 110(1-3), 1-23. https://doi.org/10.1016/j.schres.2009.03.005

Trinidad, D. R., \& Johnson, C. A. (2002). The association between emotional intelligence and early adolescent tobacco and alcohol use. Personalinity and Individual Differences, 32, 95105.

Wölwer, W., Brinkmeyer, J., Riesbeck, M., Freimüller, L., Klimke, A., Wagner, Möller, H.J., Klingberg, S.; Gaebel, W. \& German Study Group on First Episode Schizophrenia (2008). Neuropsychological impairments predict the clinical course in schizophrenia. European Archives of Psychiatry and Clinical Neuroscience, 258 Suppl 5, 28-34. https://doi.org/10.1007/s00406-008-5006-2 